FACTA UNIVERSITATIS

Series: Mechanical Engineering Vol. 15, N ${ }^{\mathrm{o}} 3$, 2017, pp. 353 - 366

https://doi.org/10.22190/FUME170928019B

Original scientific paper

\title{
BICYCLE HELMET DESIGN AND THE VIRTUAL VALIDATION OF THE IMPACT, AERODYNAMICS AND PRODUCTION PROCESS
}

\author{
UDC $539.3+533.6+621.7$
}

\section{Bojan Boshevski, Ile Mircheski}

Ss. Cyril and Methodius University in Skopje (UKIM),

Faculty of Mechanical Engineering, Republic of Macedonia

\begin{abstract}
This paper presents the development process of a bicycle helmet through individual research, creation, presentation and analysis of the results of the most important product development stages. The quality of the development and manufacturing process of the protective equipment for extreme sports is an imperative for a successful product and its flawless function. The design of the bicycle helmet is made following the rules of the design in order to create a well-founded and functional product. After creating design sketches, a virtual prototype was developed in "SolidWorks" using the required ergonomic dimensions. 3D printed model of the human head with adapted ergonomic dimensions and the designed bicycle helmet was developed in order to verify the applied ergonomic measures. The virtual model will be used as an input in the finite element analysis of the helmet impact test based on the EN1078 standard and the aerodynamic simulations executed in "SolidWorks Simulation and Flow Simulation", for verification of the impact and aerodynamic properties. Virtual testing of aerodynamic features and the ability of the bicycle helmet to allow ventilation of the user's head indicate that the helmet performs its function in the desired way. Also, the virtual prototype will be used for the production process simulation in "SolidWorks Plastics" in order to analyze the production of the bicycle helmet. The polycarbonate helmet outer shell is subject to a number of simulations for the sake of analyzing the production process in order to obtain the desired characteristics of the polycarbonate outer shell and to avoid the disadvantages that occur in the manufacturing process. The main goal of this paper is to develop a safety bicycle helmet with improved ergonomic, validation of impact, aerodynamic characteristics and production process in order to produce a high quality product for mass use.
\end{abstract}

Key Words: Design, Bicycle Helmet, Ergonomics, 3D Printing, Virtual Testing

Received September 28, 2017 / Accepted November 15, 2017

Corresponding author: Ile Mircheski

Ss. Cyril and Methodius University, Faculty of Mechanical Engineering, Karpos II bb, 1000 Skopje, Macedonia

E-mail: ile.mircheski@mf.edu.mk 


\section{INTRODUCTION}

Both proper design and protective equipment production for extreme sports form the basis for the given purpose of this type of sports equipment, that is, the user's protection and avoidance of injury with or without lasting consequences. The most important difference, if it comes to the point when the supplementary protective equipment needs to fulfill its purpose, is whether the user of the equipment benefits from its use in a given situation or the equipment does not fully and properly perform its function. The second most important factor in the use of protective equipment is the possibility for the user to make the most of the functions that are foreseen in the design and production. At the same time, the additional equipment should not cause a decrease in the physical performance of the user himself; on the contrary, it should enable an uninterrupted activity performance in addition to increasing the possibility to achieve a better desired performance. This paper synthesizes the designing process and the virtual production testing of this product in order to enable construction and optimization of those product's key features that lead to a successful product. Using the designers' approach and sketching, the final version of the bike helmet is created and converted to a 3D model using software for virtual prototyping. Simulations for testing and verification of the product's impact and aerodynamic functioning in use are made in a virtual environment. After verification of the product's aerodynamic features, another simulation is carried out; only this time the outcome is used for optimization of the helmet's first layer, the outer shell, the surface that receives the first impact and is responsible for the overall reaction of the bicycle helmet after the impact.

\section{LITERATURE REVIEW}

The most common injuries in cycling and sport activities are head ones. The protective safety equipment is a bicycle helmet. Safety is one of the most important factors when it comes to protective helmets used in sports, industry, etc. The paper [1] aims at improving the protective characteristics of the bicycle helmets, namely, to improve especially energy absorption of the liner foam in the bicycle helmet. The study is focused on finding a replacement material for EPS liner foam with improved energy absorbing characteristics. The impaxx energy absorbing foam is used; it presents a strong potential in overcoming such problems of EPS foam. This study has carried out the finite element analysis of the helmet impact test using LS-DYNA software. The studies $[2,3,4]$ presented assessment of a helmet with dual layer liner based on the shock absorbing test. The FEM is used in analyzing of helmet impact using LS-DYNA software. The simulation of the helmeted head form drop test is implemented in order to determine a structure of the liner that reaches high effective protection and to design helmet models with single and dual liners.

The paper [5] compares the results of three virtual drop tests of the protective equipment produced of three completely different types of plastic and the results of the stress and deformation values. The inspiration for writing the paper [5] comes from a large number of injuries induced in rugby matches. One of the most common injuries in this sport is the brain concussion, which occurs as a result of collision between two or more rugby players. The purpose of this research is to determine the influence of the velocity against the player's protective helmet. In order to check the helmet's performance, testing with a pneumatic linear impact system, rugby helmet and all the necessary parameters that make this testing 
reliable is conducted. The experiment has eighteen phases and in each new phase, a new rugby helmet is used and tested three times. The results obtained from the experiment are used as inputs in injury simulation of a finite elements human brain model. After the test, it was concluded that the collision velocity between players is a significant factor and that it is necessary to design a rugby helmet with a higher impact speed tolerance.

The human head is one of the most vulnerable parts of our body and because of this, it is necessary to carry a protective helmet while riding a motorcycle. The purpose of the paper [6] is to create a more functional and protective design of a motorcycle helmet, with all the necessary characteristics of a supreme helmet with top quality, including proper head ventilation as one of the most important features. In order to properly construct this type of protective equipment, a database that contains information on the effect of the overall design on all the necessary features that the helmet should have, has been created. By using this database and determining the most significant subsystems and their separation to the smallest details which are linked and associated with the subsystems, few sketches for motorcycle designs have been created. Using this method with separation of subsystems, then joining them together again, allows a creation of a design that meets all the necessary requirements.

In order to improve the homologation process of protective sports helmets the paper [7] develops a new method for angled impact testing. Besides the development of this testing method, the complete testing process, and an opportunity for making cheap, reliable, easy to use test equipment and the criteria by which one helmet would be given a certificate that approves its use are explained in this paper.

The criteria that are necessary for optimizing the protection of the head with the help of helmet are created and improved. A head model created from finite elements is subject to virtual testing of impact in order to obtain the impact values in $\mathrm{kPa}$ and $\mathrm{mJ}$, that cause minimal or severe head injury. After the impact a testing is done, helmets are placed on the tested model of the human head and through a virtual simulation, an assessment of the ability to protect the head in relation to the previously set criteria for head injury is made, resulting in the possibility of creating a better protective equipment for the head [8].

There are many ways of discovering the tendency of a model towards turbulences. Because of this, the paper [9] presents a comparative analysis for detection of the most appropriate model for the helmet turbulence analysis. In order to determine the air flow characteristics of a helmet, 2D virtual simulation with a simplified model of a human head wearing a helmet is conducted. The results of this simulation do not match with the numerical results obtained from the turbulence model. To find out where the problem arises the 3D simulation of a similar simplified model is made. With the obtained results from the 3D simulation, the turbulence model which gives the most reliable results regarding the experimentally obtained results is determined.

In the papers $[10,11]$ are investigated the aerodynamic improvements of applying a truncated airfoil shape with a trailing edge modification to a helmet design. SolidWorks Flow Simulation is used to evaluate the aerodynamic forces. A common production helmet design is progressively truncated to determine the optimal truncation length and the effect of multiple trailing edge modification is tested. Scale models of the final improved design and the production helmet were tested in the wind tunnel to verify the computational results. 
The goals in this paper are the design process, ergonomic analysis, the virtual validation of impact test, aerodynamic verification and production testing of the bicycle helmet in order to enable construction and optimization of the product's key features which lead to a successful product.

\section{CONCEPTUALIZATION OF THE BiCYCLE HELMET}

The creation of a functional and well-founded product starts with design development through sketches shown in the next page, Figs. 1, 2 and 3. This phase is very important for the product because it includes an elaborative design as well as functional characteristics which need to be compatible with the product's aesthetics, i.e. to ensure that the proper product's functioning is synthesized with the aesthetical value. It is vital to ensure balance between the design of the product and its functionality in this type of products. In Fig. 1 is shown a sketch in perspective for the bicycle helmet and in Fig. 2 is shown a sketch in multiple views. Fig. 3 illustrates the placement of the ventilation openings on the helmet's polycarbonate shell and the airflow in and around the helmet. In order to analyze the airflow inside and around the bike helmet, section 6.2 presents airflow verification. For this type of product, there should be a balance between the functional characteristics of the product and its design. The functional characteristics should not be neglected because of the design and vice versa. However, not focusing on the design could lead to an unreliable and poor quality product; therefore, creating a safe product to use is the most important task. 3D CAD model and ergonomic verification of the helmet are shown on Fig. 4. This prototype is made based on the previously created design sketches, made by using software for parametric modeling ,SolidWorks“. 3D scanned model of a male head in real ergonomic dimensions, which meets the designing standards for this type of protective equipment is used as a base for 3D modeling of the designed bicycle helmet for mountain biking. The virtual 3D model is created by using an advanced surface modeling technique available in the 3D modeling software since this design cannot be created by means of simple modeling tools.

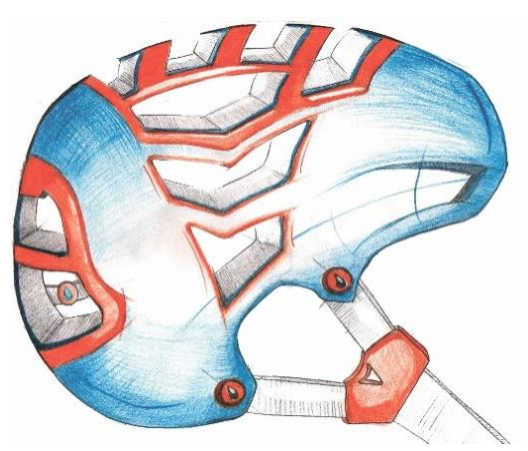

Fig. 1 Product design sketch in perspective

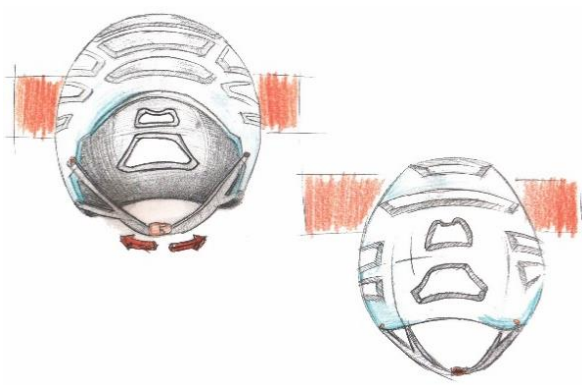

Fig. 2 Product design sketch in multiple views 


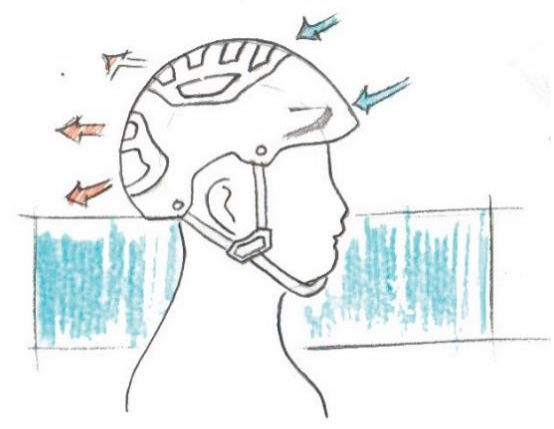

Fig. 3 Bicycle helmet air circulation sketch

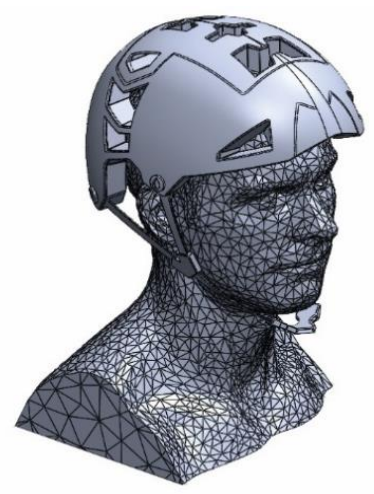

Fig. 4 Virtual model of 3D scanned head and designed bicycle helmet

\section{APPLIED ERGONOMICS IN THE DESIGN AND VALIDATION OF THE ERGONOMIC PROPERTIES WITH RAPID PROTOTYPING ON 3D PRINTER}

The ergonomics is an important issue in the product designing process. The body sizes the smallest and biggest representative of the selected users' population direct influence on the product's dimensions. With the use of necessary and exact ergonomic measures shown in Fig. 5, the development process would result in a potentially safe product. The creation of the product which is safe to use depends not only on the ergonomics itself, but also on the other design and developmental processes. The ergonomic measures used for designing this protective bicycle helmet are the key measures of the men's head in the range of $95^{\text {th }}$ percentile. This percentile is chosen because the product will be more universal in terms of covering a bigger group of users without changing the product's safety qualities. As a starting point for this product design a 3D scanned male head in real scale, shown in Fig. 6 is used. Verification of the designed virtual model ergonomic properties is accomplished by creating a 3D printed model of the bike helmet, positioned on the previously 3D scanned male head, scaled down together to allow a flawless printing process. The completed 3D printed prototype of the bicycle helmet positioned on the male's head, shown in Fig. 7, represents the applied ergonomics and its accuracy.

\section{THE DESIGN OF BiCyCle HeLMET AND 3D Modeling}

The aesthetic values of the bicycle helmet design are created and developed by using guidelines enclosed in functionalism. In this case, it is essential that the form follows the function. The basic inspiration for the design shape comes from the aerodynamic qualities of the rain drop, which represents a conjunction between naturalism and functionalism. Perforations on the bicycle helmet are minimized, and because of their correct placement, they allow proper air circulation and reduce the risk of helmet's shell weakening because of their position. 


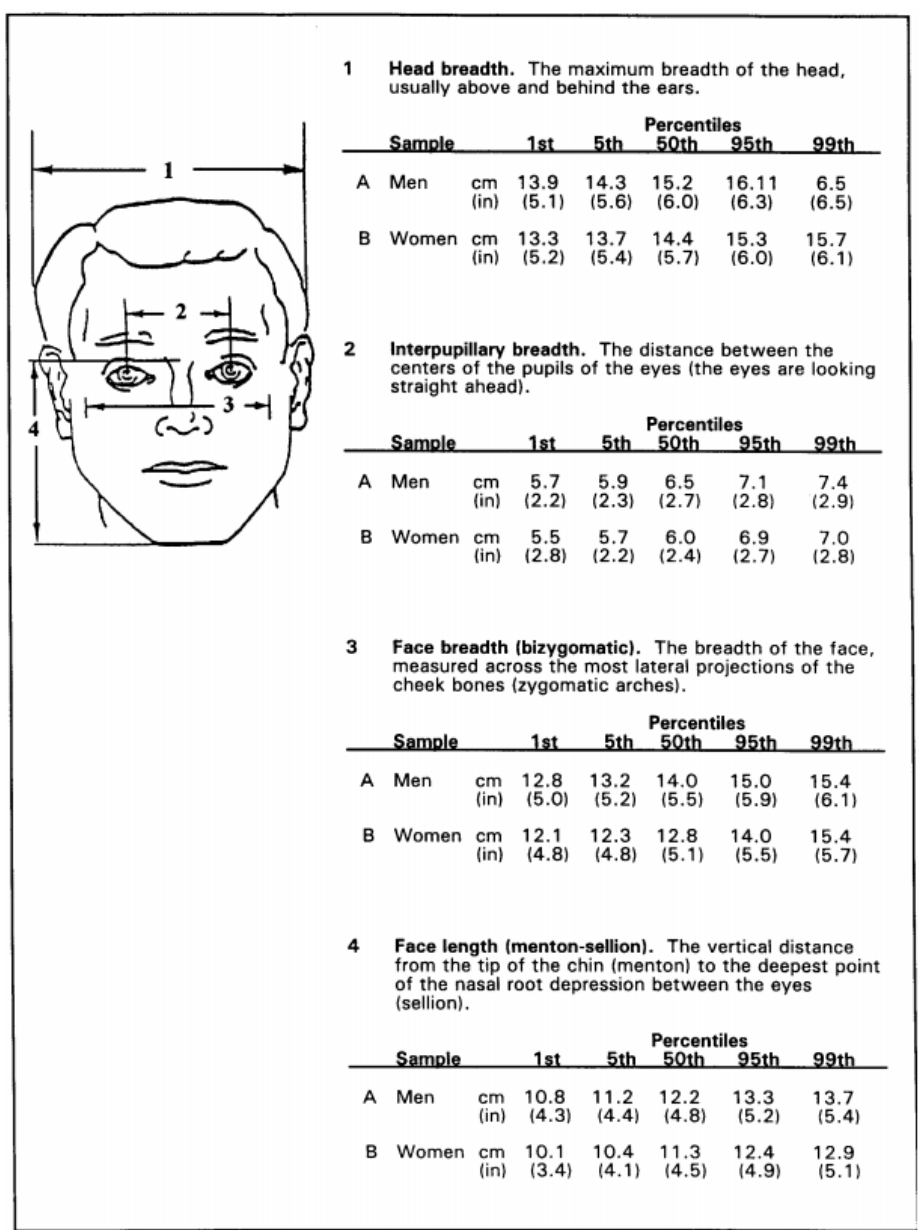

Fig. 5 Applied ergonomic measures [12]

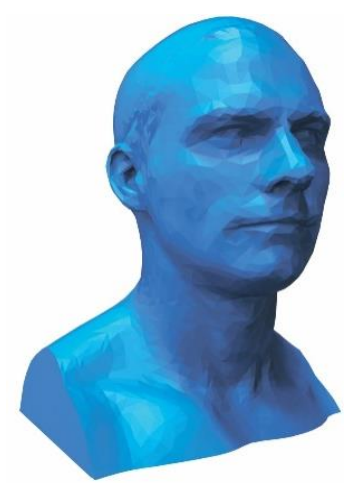

Fig. 6 3D scanned human head

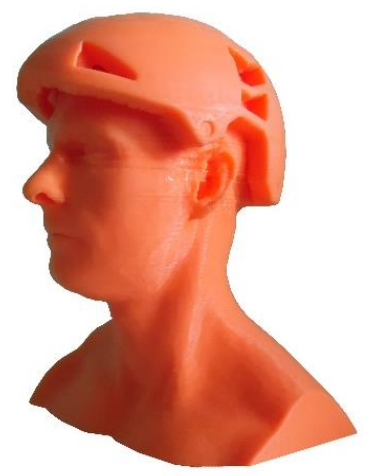

Fig. 7 3D printed model 
The elements for fixing the straps, the plastic parts for joining the straps together and the parts of the strap buckle are minimalistic and in tone with the colors and the shape used for the design of the helmet's shell. Figs. 8, 9, 10 and 11 show the bicycle helmet design and represent the style that this design belongs to.

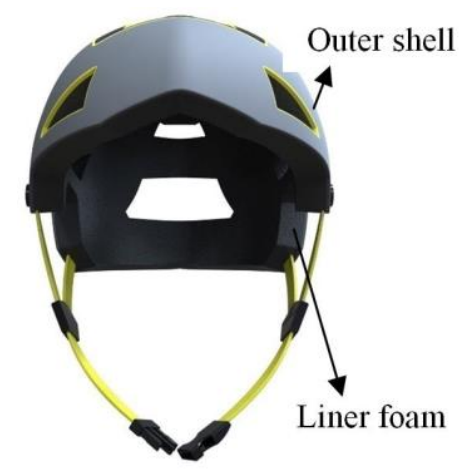

Fig. 8 Bicycle helmet front view

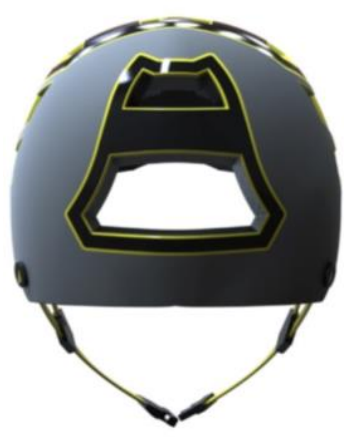

Fig. 10 Bicycle helmet rear view

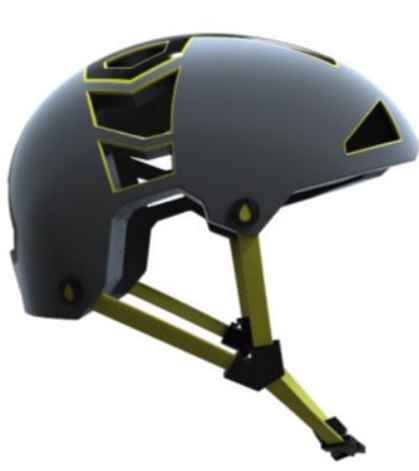

Fig. 9 Bicycle helmet side view

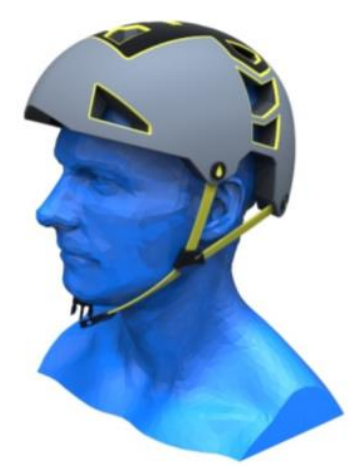

Fig. 11 Bicycle helmet on the 3D scanned head model

\section{VIRTUAL TESTING OF THE BICYCLE HELMET}

During the product development process, the phase in which the product's qualities are simulated and verified is unavoidable. This part of the development process is very important for the product and its progress. In this process phase it is possible to realize the characteristics of the product which are going to be kept, and those that will have to be modified in order to optimize the helmet's performance and its manufacturing in terms of efficiency, effectiveness and economic justification. This paper contains examination and verification of the impact and aerodynamic features of the designed bicycle helmet, when the bicycle helmet is in an active (riding) position, a passive (riding) position and in a simulation of an optimized manufacturing process of the bicycle helmet shell made of polycarbonate. 


\subsection{Finite element analysis of the helmet impact test}

The impact tests should be performed in order to confirm the bicycle helmet protection and satisfy the helmet safety standards. The basic bicycle helmet is composed of outer shell and liner foam. The outer shell protects the head from impact and injures. The outer shell is usually made of polycarbonate (PC) or acrylonitrile butadiene styrene (ABS). In the case study, for outer shell is used PC material with the following material properties: Young's modulus of $2440 \mathrm{MPa}$, density of $1400 \mathrm{~kg} / \mathrm{m} 3$ and Poisson's ratio of 0,4 . Expanded polystyrene (EPS) foam is material with a good energy absorption characteristic which is used for bicycle helmet. The protective liner foam is made of EPS foam. The thickness of the PC outer shell is $2,5 \mathrm{~mm}$ and the liner thickness in this case study is $25 \mathrm{~mm}$. The material properties used in this case study for EPS liner foam are: Young's modulus of $40 \mathrm{MPa}$, density of $1100 \mathrm{~kg} / \mathrm{m} 3$ and Poisson's ratio of 0,1 . The flat anvil is a rigid body. The distance between the helmet and the anvil in the beginning is $1,5 \mathrm{~m}$ as initial condition. The meshed model with tetrahedron elements is shown in Fig. 12 and prepared to be used in the FEA. The number of total finite elements is 86964 . The bike helmet FE model drops onto a flat anvil plate under gravity and load from body weight from $120 \mathrm{~kg}$.

The virtual testing is performed with the finite element analysis (FEA) using SolidWorks Simulation software package. The FEA is used to obtain stress field and results of von Mises stress field for outer shell from the bike helmet. Fig. 13 shows the results from the von Mises stress field for the bike helmet. The maximum von Mises stress for the PC outer shell is 44,5 MPa. The yield strength for PC material is $60 \mathrm{MPa}$ and the outer shell is in elastic range. In this case study the cyclist will be protected from head injuries since the bicycle helmet materials will be in elastic range.

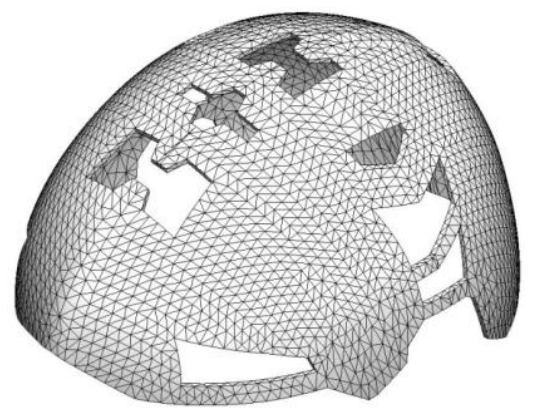

Fig. 12 The meshed model of bicycle helmet

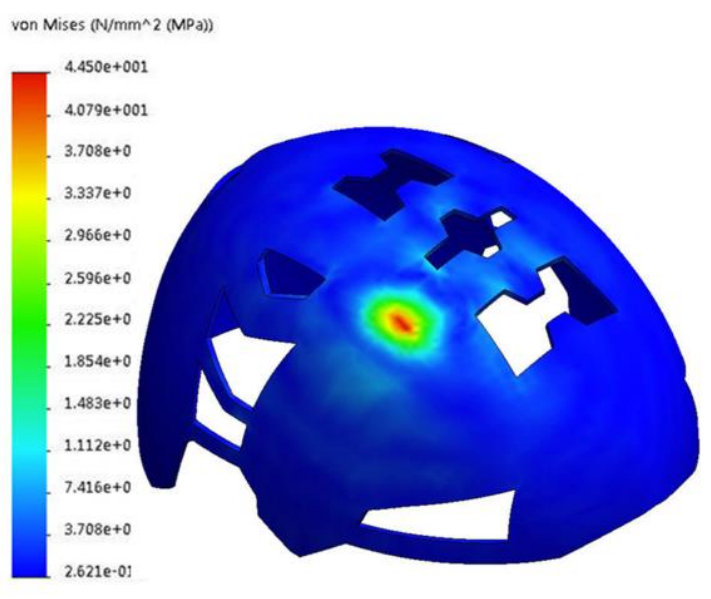

Fig. 13 FEA of the bicycle helmet under impact test

\subsection{Examination and verification of aerodynamic properties}

The most important feature of bicycle helmets is their aerodynamic; therefore, it is important to examine and verify its functioning. Testing of aerodynamic properties is 
usually done before the serial production begins in order to determine the correctness of the design and its proper functioning. Virtual testing for the bicycle helmet design is performed using "SolidWorks Flow Simulation".

The input values for initial and boundary conditions used for the virtual and experimental testing of the aerodynamic properties are: input velocity of $25 \mathrm{~km} / \mathrm{h}$, atmospheric pressure of $101325 \mathrm{~Pa}$, earth acceleration of $9.81 \mathrm{~m} / \mathrm{s}^{2}$, and ideal walls of the air tunnel. In Fig. 14 for the first virtual testing are presented the results for the velocity of air around of helmet in every point and airflow lines. The helmet is positioned on a human head in a vertical position in order to properly display the velocity, airflow lines and the ventilation of the head outside the bike helmet. The results from the presented flow charts for the first virtual testing reveal a large turbulence after contact with helmet. The airflow in the first virtual testing is more complex and requires a longer time to stabilize.

In the second virtual testing, shown in Fig. 15, the helmet is also positioned on the human head and the input values are identical to the first virtual testing, but this time the head is set at an angle of $30^{\circ}$ in relation to the lower plane, representing an active bicycle riding position, where the helmet aerodynamics should fully emphasize its performance. The difference between the passive position (first virtual testing) of the helmet and the active position (second virtual testing) of the helmet can be seen in Fig. 15 and the airflow diagram. The diagram in the second virtual testing shows faster stabilization of the velocity, lower turbulence after contact with helmet and improved ventilation and air circulation in the helmet.

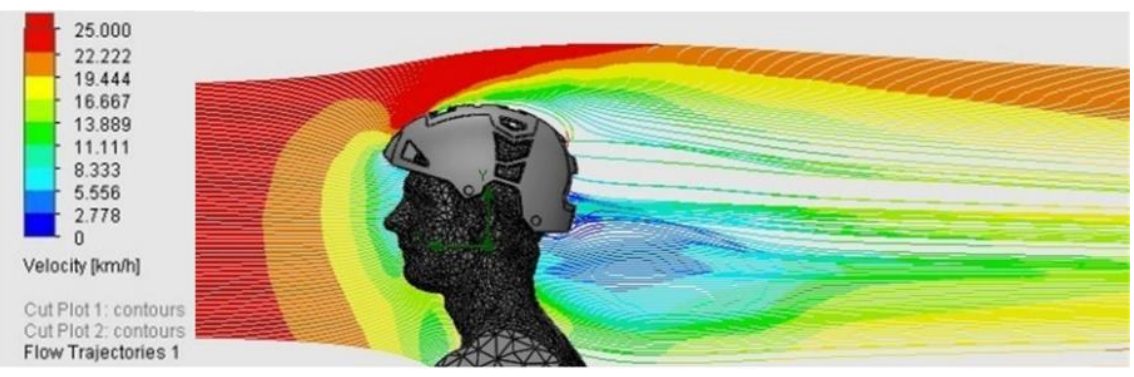

Fig. 14 Airflow lines and velocity diagram for a vertically placed bicycle helmet

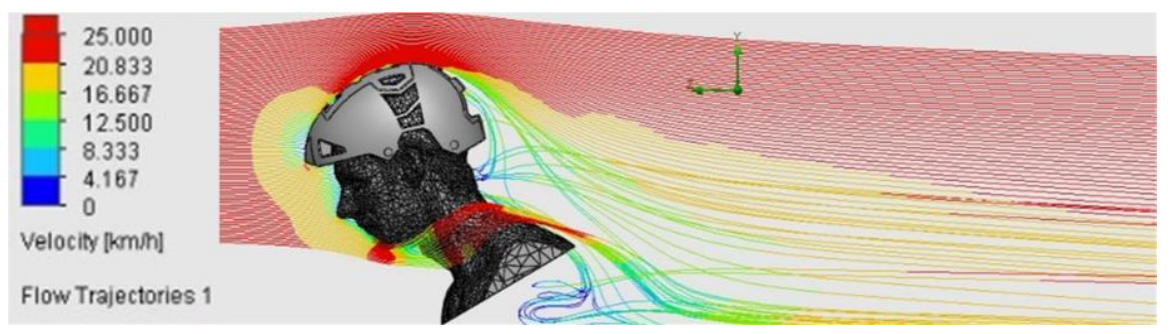

Fig. 15 Air flow and velocity diagram for inclination of human head at an angle of $30^{\circ}$

In addition to the aerodynamics of the bicycle helmet, the above mentioned entrance of the air into the helmet or the ventilation of the user's head, which is made possible by positioning the ventilation openings in the precisely determined places are very important in order to enable constant air circulation. The air circulation in the bicycle helmet is shown in Fig. 16. 


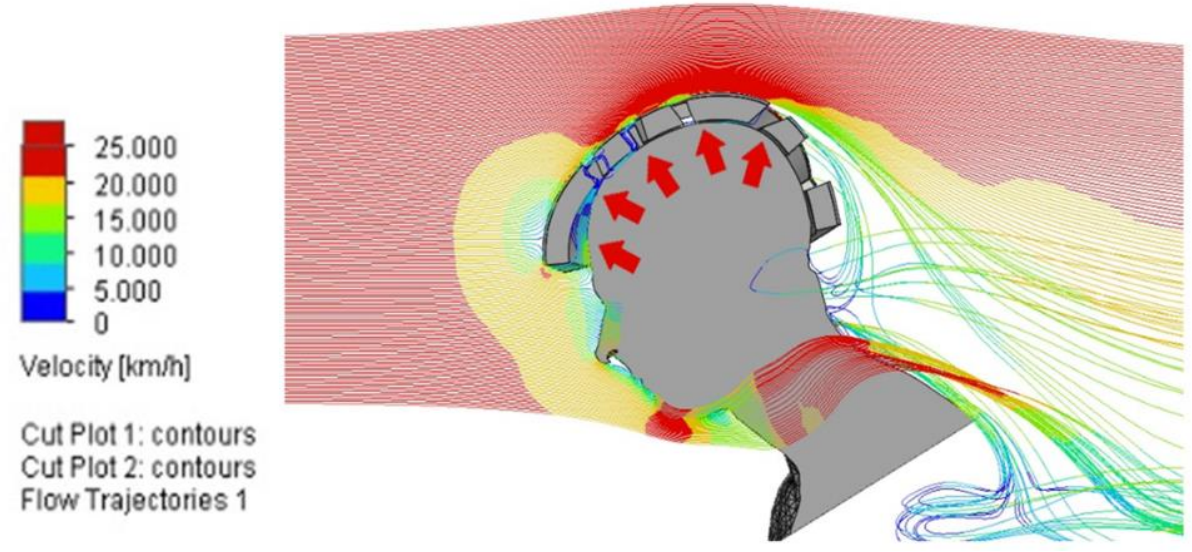

Fig. 16 Air circulation inside the bicycle helmet

In order to verify the results from the virtual testing of aerodynamics the bicycle helmet was tested in a wind tunnel and experimentally verified with the equipment shown in Fig. 17. The wind tunnel at the Faculty of Mechanical Engineering in Skopje and the measurement equipment were used to perform verification. The result for the airflow lines between the virtual testing and the experimental one with the same initial and boundary conditions are compared and the airflow lines are in correspondence as presented in Fig. 18.

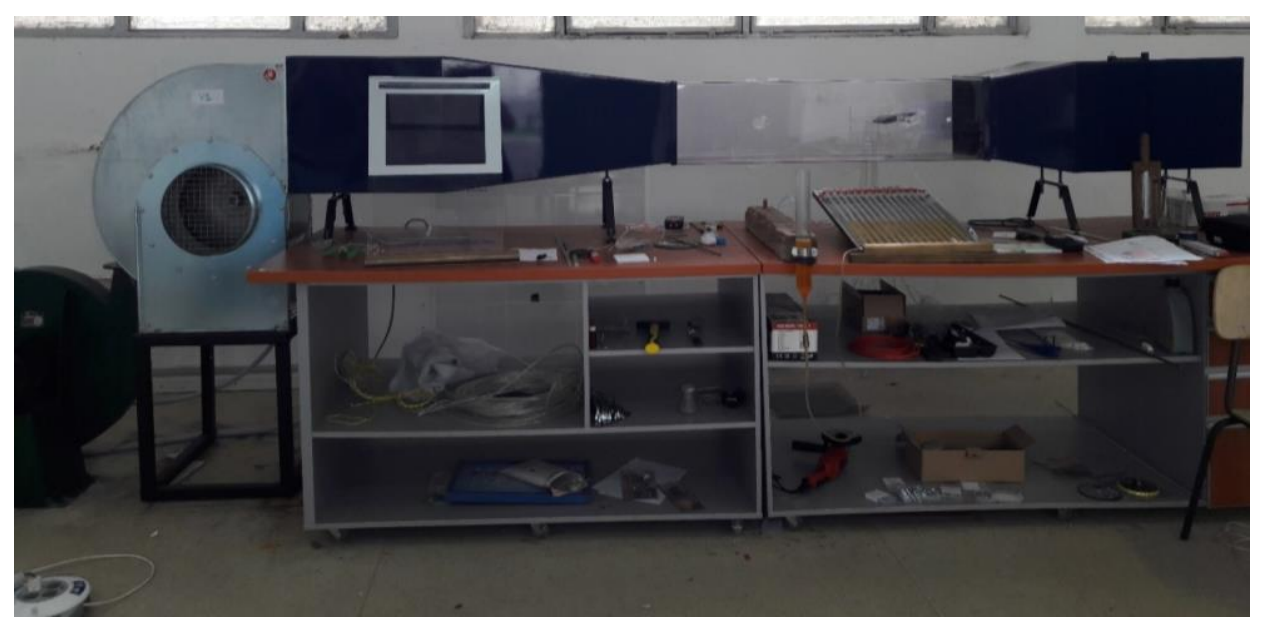

Fig. 17 Wind tunnel used for experimental testing 
a)

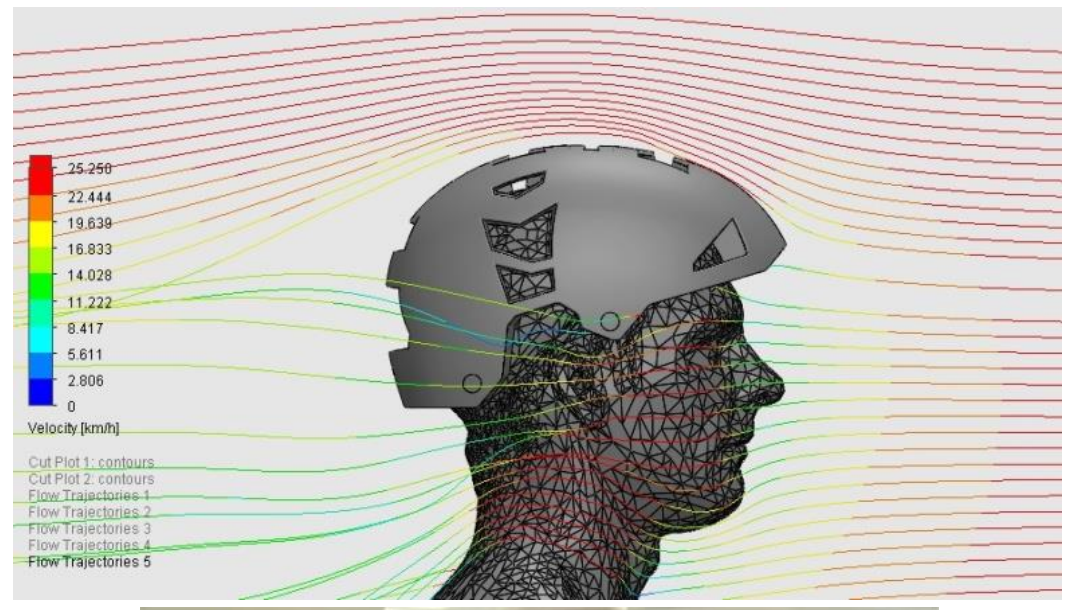

b)

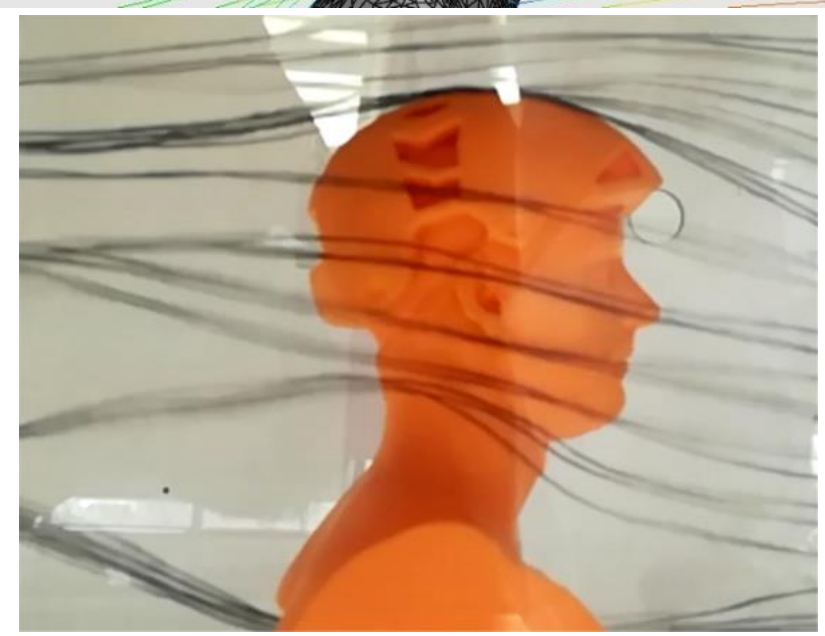

Fig. 18 The airflow lines for a vertically placed bicycle helmet obtained by: a) virtual testing and $b$ ) experimental testing

\subsection{Virtual testing of the production process of the bicycle helmet outer shell}

Virtual testing of a plastic part injection molding process is an economical and effective option when it comes to larger and more complex products. Instead of doing physical production testing with certain values of the inputs, the testing in a virtual environment allows combining values in a faster and simpler way in order to obtain mutually compatible input values that will result in a high quality and feasible product. Virtual testing of the bicycle helmet production process was made using SolidWorks Plastics. In order to obtain results that are credible and applicable in reality, more virtual testing was done by changing the input values and taking into account several parameters that the quality and endurance of the bicycle helmet depend on. The parameters that were analyzed are: the position of the injection gates, sink marks, the residual stress in the part, the total displacement of the part after the process and the weld lines. Those parameters have a significant impact on the 
design and aesthetic of the bike helmet. The input values that have been changed in order to keep the residual stress within their safe limit are: the number of injection gates (four in the last virtual test) symmetrically positioned with a $3 \mathrm{~mm}$ diameter nozzle and shown in Fig. 19; the temperature of the mold is increased to $95{ }^{\circ} \mathrm{C}$ and the packing time is set to 18 seconds. With the change of the input values, the desired values of the analyzed parameters are obtained. Sink marks are shown in Fig. 20, which have maximum value of $0.0601 \mathrm{~mm}$; they occur in the safe areas, that is, in the helmet areas that are not critical for the user's safety. The residual stress shown in Fig. 21 appears in its proportional limit with a maximum value of $17.89 \mathrm{MPa}$ and because the stress is lower than the maximum limit value of the material's yield strength, the bicycle helmet shell would not undergo major plastic deformations. The total deformation of the plastic part, shown in Fig. 22, is $0.8337 \mathrm{~mm}$. due to the size of the part, is acceptable and negligible. The weld lines which are shown in Fig. 23 are critical points where the material collides and where the part is the weakest; they are in the areas that would not be affected under the influence of mechanical forces on the helmet, while the helmet is on the user's head. With the obtained results, the virtual testing of the production process is fully optimized and the virtual testing is successful.

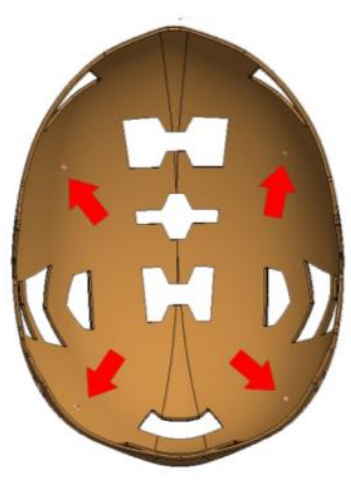

Fig. 19 Positions of the injection nozzle

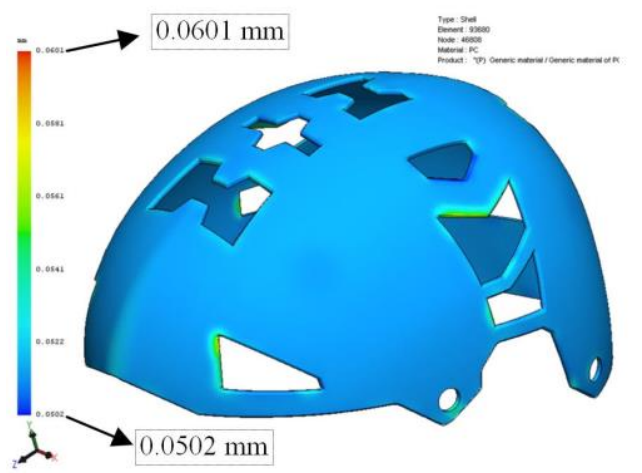

Fig. 20 Sink marks of the PC shell

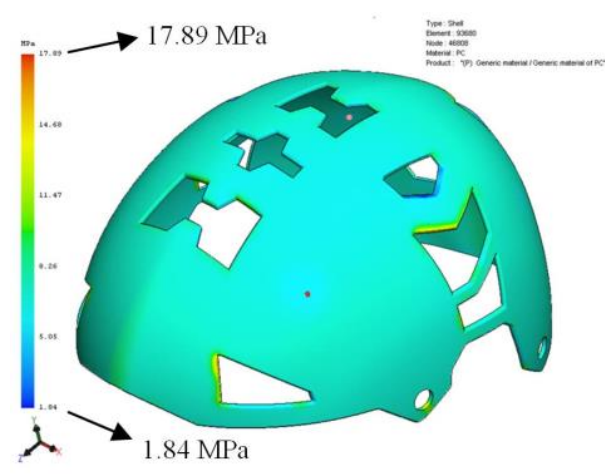

Fig. 21 Residual stress at post-filling end of PC shell

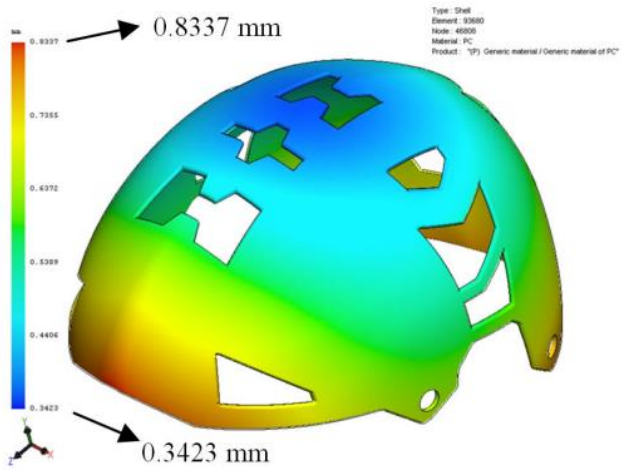

Fig. 22 Total stress displacement of the PC shell 


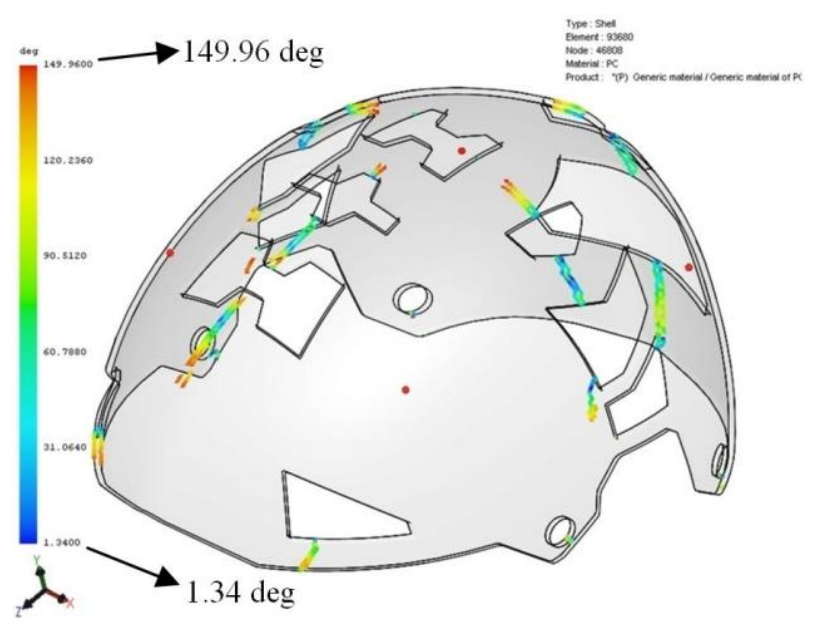

Fig. 23 Weld lines of the PC shell

\section{CONCLUSION}

This paper shows the basic developmental process of a product bicycle helmet. The main objective of this paper is to present the process of a protective bicycle helmet development which will not end up with just an idea and a virtual 3D model, but will continue with the rest of the developmental phases. The ergonomic characteristics of the designed bicycle helmet are validated with a 3D printed prototype in real dimensions. The bicycle helmet impact test model used SolidWorks Simulation while the design was based on virtual testing. This study indicates that the design and the virtual validation of the helmet impact test are satisfied; it also predicts safety of helmet during impacts. Example for this type of limitation is the airflow around and in the designed bicycle helmet, which in this case represents the imperative for a successful product. Because of this, it is very important to position the ventilation openings correctly and this is only possible with previously conducted virtual air flow testing. Besides the helmets' design, the virtual testing for the helmet's performance and its outer shell manufacture are presented in order to point out that it is very important to realize and overcome the limitations which appear and affect the development process and the helmet's design. With the use of modern computer technology and the right parameters we can get reliable results as well as a realistic representation of the function of the developed and tested product. Using the computer technology and software for modeling, virtual testing and the result analysis, the time required for designing and preparation of the manufacturing process is significantly reduced, which, in its turn, reduces the costs indirectly. 


\section{REFERENCES}

1. Tso-Liang, T., Cho-Chung, L., Chien-Jong, S., Van-Hai N., 2013, Design and Analysis of Bicycle Helmet with Impaxx Foam Liner, Advanced Materials Research, 706-708, pp. 1778-1781.

2. Tso-Liang, T., Cho-Chung, L., Van-Hai, N., 2013, Design of Dual Layer Liner with Deformable Semi-Spherical Convex for Bicycle Helmet, Applied Mechanics and Materials, 284-287, pp. 681-686.

3. Tso-Liang, T., Cho-Chung, L., Van-Hai, N., 2016, Assessment of a bicycle helmet liner with semispherical cones, Proc IMechE Part L: J Materials: Design and Applications, 230(1), pp. 344-352, DOI: $10.1177 / 1464420715569290$

4. Tso-Liang, T., Cho-Chung, L., Van-Hai, N., 2014, Innovative design of bicycle helmet liners, J Materials: Design and Applications, 228(4), pp. 341-351.

5. Post, A., Oeur, A., Hoshizaki, T. B., Gilchrist, D. M., 2013, The influence of velocity on the performance range of american football helmets, Report, Human Kinetics University of Ottawa, Ottawa, Canada, School of Mechanical \& Materials Engineering, University College Dublin

6. Zhihua, W., Peter, R. N., Childs, D., Kak, L., 2013, Application of an effects database in idea generation approach for helmet design, Report, Department of Mechanical Engineering, Imperial College London, London, UK, Department of Innovation Design Engineering, Royal College of Art, London, UK.

7. Halldin, P., Kleiven, S., 2013, The development of next generation test standards for helmets, Report, Royal Institute of Technology, Stockholm, Sweden.

8. Deck, C., 2013, Model based head injury criteria for head protection optimization, Report, University Strasbourg \& CNRS, Strasbourg, France.

9. Shishodia, B. S, Sanghi, S., Mahajan, P. A., 2013, Comparative study of turbulence models performance for the study of air flow in helmets, Report, Applied Mechanics Department Indian Institute of technology Delhi, New Delhi, India.

10. Bradford, W. S., Jenkins, E. P., 2011, Aerodynamic bicycle helmet design using a truncated airfoil with trailing edge modifications, Proceedings of the ASME 2011 International Mechanical Engineering Congress, Denver, Colorado, USA.

11. Rodrigue-Millan, M., Tan, L. B., Tse, K. M., Lee, H. P., Miguelez, M. H., 2017, Effect of full helmet systems on human head responses under blast loading, Material \& Design, Vol. 117, pp. 58-71, https://doi.org/10.1016/j.matdes.2016.12.081.

12. Human factors standardization Subtag, 2000, Human engineering design data digest, Washington, pp. 79-82. 